\title{
Pseudoscalar meson transition form factors *
}

\author{
A.-Z. Dubničkováił ${ }^{\dagger}$ S. Dubnička² ${ }^{2}$, G. Pancheri ${ }^{3}$ and R. Pekárik ${ }^{2}$ \\ ${ }^{1}$ Dept. of Theor. Physics, Comenius Univ., Bratislava, Slovak Republic \\ ${ }^{2}$ Inst. of Physics, Slovak Academy of Sciences, Bratislava, Slovak Republic \\ ${ }^{3}$ Lab. Nazionali di Frascati dell' INFN, via E. Fermi 40, I-0044 Frascati (Roma), Italy
}

\begin{abstract}
Present-day experimental and phenomenological situation about pseudoscalar $\left(\pi^{0}, \eta, \eta^{\prime}\right)$ meson transition form factors is briefly reviewed. Necessity of more sophisticated behaviours in time-like region of these form factors is emphasized. Four-resonance unitary and analytic model of the pseudoscalar meson transition form factors is constructed, which describes well all existing experimental information.
\end{abstract}

\section{INTRODUCTION}

Nowadays we know, that hadrons have a finite size, which in electromagnetic (EM) interactions is revealed as the EM structure of hadrons, phenomenologically to be described by one or more (it depends from the spin of hadron under consideration) functions of one variable (the four-momentum transfer squared $t=q^{2}=-Q^{2}$ of a virtual photon), called the EM form factors (FF's). If in the vertex with three lines appear one virtual photon and two identical strongly interacting particles, one speaks about the elastic EM FF's of hadron. If there is one virtual photon and two different

\footnotetext{
${ }^{*}$ Contribution presented at the PHOTON'03, April 7-11, 2003, Frascati (Roma), Italy

†e-mail: dubnickova@fmph.uniba.sk
} 
hadrons (or one hadron and one real photon) we speak about the transition FF's. A subgroup of the latter represent the pseudoscalar meson transition FF's, describing any $\gamma^{*} \rightarrow \gamma P$ transition, where $P$ can be $\pi^{0}, \eta$ and $\eta^{\prime}$.

Unlike the elastic meson and baryon EM FF's, the pseudoscalar meson transition FF's are not well elaborated till now, though there are special physical problems in which they play an important role. For instance, there is no evaluation of the $e^{+} e^{-} \rightarrow$ $\gamma P$ contributions to the muon anomalous magnetic moment (in connection with its precise measurement at the E-821 experiment in BNL) just due to the absence of a reliable behaviour of the corresponding transition FF's in the time-like region.

In this contribution we solve the latter problem by a construction of unitary and analytic models of the pseudoscalar meson transition FF's, which reproduce existing experimental data in space-like and time-like region quite well.

\section{PRESENT THEORETICAL NOTIONS}

There is a single $\mathrm{FF}$ for each $\gamma^{*} \rightarrow \gamma P$ transition to be defined by a parametrization of the matrix element of the EM current $J_{\mu}^{E M}=2 / 3 \bar{u} \gamma_{\mu} u-1 / 3 \bar{d} \gamma_{\mu} d-1 / 3 \bar{s} \gamma s$

$$
\left\langle P(p) \gamma(k)\left|J_{\mu}^{E M}\right| 0\right\rangle=\epsilon_{\mu \nu \alpha \beta} p^{\nu} \epsilon^{\alpha} k^{\beta} F_{\gamma P}\left(q^{2}\right)
$$

where $\epsilon^{\alpha}$ is the polarization vector of the photon $\gamma, \epsilon_{\mu \nu \alpha \beta}$ appears as only the pseudoscalar meson belongs to the abnormal spin-parity series.

A straightforward calculation of $F_{\gamma P}\left(Q^{2}\right)$ behaviour in QCD is impossible and one can obtain Brod81 in the framework of PQCD only the asymptotic behaviour

$$
\lim _{Q^{2} \rightarrow \infty} Q^{2} F_{\gamma P}\left(Q^{2}\right)=2 f_{P}
$$

where $f_{P}$ is the meson weak decay constant.

The behaviour of $F_{\gamma P}\left(Q^{2}\right)$ for $Q^{2} \rightarrow 0$ can be determined from the axial anomaly in the chiral limit of QCD

$$
\lim _{Q^{2} \rightarrow 0} F_{\gamma P}\left(Q^{2}\right)=\frac{1}{4 \pi^{2} f_{P}} \equiv F_{\gamma P}(0) .
$$

In order to describe the soft nonperturbative region of $Q^{2}$ a simple interpolation formula has been proposed by Brodsky, Lepage Brod81

$$
F_{\gamma P}\left(Q^{2}\right)=\frac{1}{4 \pi^{2} f_{P}} \cdot \frac{1}{1+\left(Q^{2} / 8 \pi^{2} f_{P}^{2}\right)}
$$


which, however, does not describe even the space-like data well. As a consequence, in the space-like region $(t<0)$ one usually fits the experimentally observed $t=-Q^{2}$ dependence of $F_{\gamma P}\left(Q^{2}\right)$ by a normalized empirical formula

$$
F_{\gamma P}(t) / F_{\gamma P}(0)=\frac{1}{\left(1-t / \Lambda_{P}\right)}
$$

where $1 / \Lambda_{P}=\left\langle r_{P}^{2}\right\rangle / 6$ is related to the size $\left\langle r_{P}^{2}\right\rangle$ of the pseudoscalar meson $\mathrm{P}$.

And in the time-like region $(t>0)$, of the well known vector-meson resonance behaviour, one is left with Breit-Wigner extension of the VMD model

$$
F_{\gamma P}(t)=\sum_{v} \frac{f_{v P \gamma}}{f_{v}} \cdot \frac{m_{v}^{2}}{m_{v}^{2}-t-i m_{v} \Gamma_{v}}
$$

which, however, is justified only at the region of resonances and generally violates the unitarity condition and the normalization.

From all mentioned above it follows that it is highly desirable to construct more sophisticated model of the pseudoscalar meson transition FF's.

\section{EXPERIMENTAL SITUATION}

There are dominating experimental data on the pseudoscalar meson transition FF's in

the space-like region Berg84,Ber91,Aha90,Gron98. However, recent measurements of the $e^{+} e^{-} \rightarrow \eta \gamma, \pi^{0} \gamma$ processes at VEPP-2M in Novosibirsk Acha99 around $\phi$-meson supplemented older data Die80,Druz84,Dol89,Vik80 at lower energies and they all provide a possibility to carry out a serious analysis of the corresponding FF's. So, at present day we have all together

- for $\pi^{0}: \quad 33$ points

- for $\eta$ : 52 points

- for $\eta^{\prime}: 59$ points.

In the next section we construct unitary and analytic model of the pseudoscalar meson transition FF's which unifies all known properties of FF's and the existing data will be utilized for an evaluation of free parameters of the model. 


\section{UNITARY AND ANALYTIC MODEL}

So, our intention is to achieve a description of all $t<0$ and $t>0$ data on the pseudoscalar meson transition FF's by one, however, distinct for $\pi^{0}, \eta$ and $\eta^{\prime}$, analytic function explicitly known on the real axis of $t$-plane from $-\infty$ to $+\infty$, respecting all properties of $F_{\gamma P}(t)$ like the asymptotic behaviour (2), the normalization (3), the analytic properties of $F_{\gamma P}(t)$, the reality condition $F_{\gamma P}^{*}(t)=F_{\gamma P}\left(t^{*}\right)$ from which it directly follows a property of the unitarity condition, that the $\operatorname{Im} F_{\gamma P}(t)$ is $\neq 0$ only from the lowest branch point on the positive real axis of $t$-plane to $+\infty$, etc.

The transition $\mathrm{FF} F_{\gamma P}(t)$ is suitable to split into two terms depending on the isotopic character of the photon

$$
F_{\gamma P}(t)=F_{\gamma P}^{I=0}(t)+F_{\gamma P}^{I=1}(t)
$$

where $F_{\gamma P}^{I=0}(t)$ can be saturated only by isoscalar vector mesons and $F_{\gamma P}(t)^{I=1}$ can be saturated only by isovector vector-mesons, whereby both sets are characterized by the photon quantum numbers.

The analytic properties of $F_{\gamma P}(t)$ consist in the assumption, that $F_{\gamma P}(t)$ is analytic

in the whole complex $t$-plane besides the cut on the positive real axis from $t_{0}=m_{\pi^{0}}^{2}$ up to $+\infty$, because there is the intermediate $\pi^{0} \gamma$ state allowed in the unitarity condition of every $\pi^{0}, \eta$ and $\eta^{\prime}$ transition FF, which generates just the lowest branch point $t_{0}=m_{\pi^{0}}^{2}$. Moreover, from the unitarity condition it follows that there is an infinite number of higher branch points on the positive real axis of $t$-plane as there is allowed infinite number of higher intermediate states in the unitarity condition of FF's under consideration. In our model we restrict ourselves to two square-root cut approximation of the latter picture. In order to fuse such analytic properties into our model we start with the following 4 resonance VMD model parametrization of (7)

$$
F_{\gamma P}(t)=\sum_{s=\omega, \phi, \omega^{\prime}} \frac{m_{s}^{2}}{m_{s}^{2}-t}\left(f_{s \gamma P} / f_{s}^{e}\right)+\frac{m_{\rho}^{2}}{m_{\rho}^{2}-t}\left(f_{\rho \gamma P} / f_{\rho}^{e}\right),
$$

and apply the normalization condition (3). However, in order to take into account the fact, that $f_{\eta}$ and $f_{\eta^{\prime}}$ (unlike $f_{\pi}$ ) are not directly measurable quantities, employing the relation for two-photon partial width

$$
\Gamma(P \rightarrow \gamma \gamma)=\frac{\alpha^{2}}{64 \pi^{3} f_{P}^{2}} m_{P}^{3}
$$


of the pseudoscalar meson $P$, one comes to a redefinition of the norm through the known partial two-photon width $\Gamma(P \rightarrow \gamma \gamma)$ in the following way

$$
F_{\gamma P}(0)=\frac{2}{\alpha m_{P}} \sqrt{\frac{\Gamma(P \rightarrow \gamma \gamma)}{\pi m_{P}}} .
$$

Then from (8) one obtains the following condition on coupling constant ratios

$$
F_{\gamma P}(0)=\sum_{s=\omega, \phi, \omega^{\prime}}\left(f_{s \gamma P} / f_{s}\right)+\left(f_{\rho \gamma P} / f_{\rho}\right)
$$

from which one can express e.g. $\left(f_{\omega^{\prime} \gamma P} / f_{\omega^{\prime}}\right)$ by all others. Substituting the latter into (8) one gets the VMD parametrization

$$
\begin{aligned}
& F_{\gamma P}(t)=F_{\gamma P}(0) \frac{m_{\omega^{\prime}}^{2}}{m_{\omega^{\prime}}^{2}-t}+ \\
+ & {\left[\frac{m_{\omega}^{2}}{m_{\omega}^{2}-t}-\frac{m_{\omega^{\prime}}^{2}}{m_{\omega^{\prime}}^{2}-t}\right] a_{\omega}+} \\
+ & {\left[\frac{m_{\phi}^{2}}{m_{\phi}^{2}-t}-\frac{m_{\omega^{\prime}}^{2}}{m_{\omega^{\prime}}^{2}-t}\right] a_{\phi}+} \\
+ & {\left[\frac{m_{\rho}^{2}}{m_{\rho}^{2}-t}-\frac{m_{\omega^{\prime}}^{2}}{m_{\omega^{\prime}}^{2}-t}\right] a_{\rho}, \quad a_{i}=\left(f_{i \gamma P} / f_{i}\right) }
\end{aligned}
$$

to be normalized automatically. In order to obtain from (12) the unitary and analytic model, we incorporate the two-cut approximation of the true analytic properties by an application of the nonlinear transformation

$$
t=t_{0}-\frac{4\left(t_{i n}^{s}-t_{0}\right)}{[1 / V-V]^{2}} ; \quad t=t_{0}-\frac{4\left(t_{i n}^{v}-t_{0}\right)}{[1 / W-W]^{2}}
$$

to the isoscalar and isovector ( $\rho$-meson) terms and subsequently also nonzero values of vector-meson widths, $\Gamma_{s} \neq 0$ and $\Gamma_{\rho} \neq 0$, are established. The effective squareroot branch points $t_{i n}^{s}$ and $t_{i n}^{v}$ include in average contributions of all higher important thresholds in both, isoscalar and isovector case, respectively, and are left to be free parameters of the constructed model. The variable $V(W)$ in $(13)$ is conformal mapping

$$
\begin{gathered}
V(t)=i \frac{\sqrt{q_{i n}^{s}+q}-\sqrt{q_{i n}^{s}-q}}{\sqrt{q_{i n}^{s}+q}+\sqrt{q_{i n}^{s}-q}} \\
q=\left[\left(t-t_{0}\right) / t_{0}\right] ; \quad q_{i n}^{s}=\left[\left(t_{i n}^{s}-t_{0}\right) / t_{0}\right]
\end{gathered}
$$

of the four -sheeted Riemann surface in $t$-variable into one $V$-plane ( $W$-plane). 
As a result of application of (13) all VMD terms in (12) first give the factorized forms

$$
\begin{aligned}
& \frac{m_{i}^{2}}{\left(m_{i}^{2}-t\right)}=\left(\frac{1-V^{2}}{1-V_{N}^{2}}\right)^{2} . \\
& \frac{\left(V_{N}-V_{i_{0}}\right)\left(V_{N}+V_{i_{0}}\right)\left(V_{N}-1 / V_{i_{0}}\right)\left(V_{N}+1 / V_{i_{0}}\right)}{\left(V-V_{i_{0}}\right)\left(V+V_{i_{0}}\right)\left(V-1 / V_{i_{0}}\right)\left(V+1 / V_{i_{0}}\right)}
\end{aligned}
$$

on the pure asymptotic term $\left(\frac{1-V^{2}}{1-V_{N}^{2}}\right)^{2}$, independent on the flavour of vector-mesons under consideration (however it depends on the isospin) and carrying just the asymptotic behaviour $|t| \rightarrow \infty \sim t^{-1}$ of the VMD model, and the so-called resonant term (the second one in (15)) describing the resonant structure of VMD terms, which however, for $|t| \rightarrow \infty$ is going out on the real constant and so, it doesn't contribute to the asymptotic behaviour of the VMD model

The subindex 0 in (15) means that still $\Gamma=0$ of all vector mesons is considered.

In order to demonstrate the reality condition $F_{\gamma P}^{*}(t)=F_{\gamma P}\left(t^{*}\right)$ explicitly, one can utilize in (15) relations between complex conjugate values of the corresponding zerowidth VMD model pole positions in $V$ (or $W$ ) plane

$$
V_{\omega_{0}}=-V_{\omega_{0}}^{*} ; \quad W_{\rho_{0}}=-W_{\rho_{0}}^{*}
$$

and

$$
V_{i_{0}}=1 / V_{i_{0}}^{*} \quad \text { for } \quad i=\phi, \omega^{\prime}
$$

following from the reality that in a fitting procedure of existing data on $F_{\gamma P}(t)$ such $t_{i n}^{s}\left(\right.$ or $\left.t_{i n}^{v}\right)$ is found that

$$
\left(m_{i}^{2}-\Gamma_{i}^{2} / 4\right)<t_{i n}^{s}, t_{i n}^{v} \quad i=\omega, \rho
$$

and

$$
\left(m_{j}^{2}-\Gamma_{j}^{2} / 4\right)>t_{i n}^{s} \quad j=\phi, \omega^{\prime}
$$

Finally, incorporating $\Gamma \neq 0$ by a substitution

$$
m_{r}^{2} \rightarrow\left(m_{r}-i \Gamma_{r} / 2\right)^{2}
$$

one comes to the unitary and analytic models of $F_{\gamma P}(t)$ in the following form

$$
F_{\gamma P}[V(t)]=\left(\frac{1-V^{2}}{1-V_{N}^{2}}\right)^{2} .
$$




$$
\begin{aligned}
\cdot & \left\{F_{\gamma P}(0) \mathbf{V}_{\omega^{\prime}}+\left[\mathbf{V}_{\omega}-\mathbf{V}_{\omega^{\prime}}\right] a_{\omega}+\right. \\
+ & {\left.\left[\mathbf{V}_{\phi}-\mathbf{V}_{\omega^{\prime}}\right] a_{\phi}-\mathbf{V}_{\omega^{\prime}} a_{\rho}\right\}+ } \\
+ & \left(\frac{1-W^{2}}{1-W_{N}^{2}}\right)^{2} \mathbf{W}_{\rho} a_{\rho}
\end{aligned}
$$

where

$$
\begin{gathered}
\mathbf{V}_{\omega^{\prime}}=\frac{\left(V_{N}-V_{\omega^{\prime}}\right)\left(V_{N}-V_{\omega^{\prime}}^{*}\right)\left(V_{N}+V_{\omega^{\prime}}\right)\left(V_{N}+V_{\omega^{\prime}}^{*}\right)}{\left(V-V_{\omega^{\prime}}\right)\left(V-V_{\omega^{\prime}}^{*}\right)\left(V+V_{\omega^{\prime}}\right)\left(V+V_{\omega^{\prime}}^{*}\right)} \\
\mathbf{V}_{\omega}=\frac{\left(V_{N}-V_{\omega}\right)\left(V_{N}-V_{\omega}^{*}\right)\left(V_{N}-1 / V_{\omega}\right)\left(V_{N}-1 / V_{\omega}^{*}\right)}{\left(V-V_{\omega}\right)\left(V-V_{\omega}^{*}\right)\left(V-1 / V_{\omega}\right)\left(V-1 / V_{\omega^{\prime}}^{*}\right)} \\
\mathbf{V}_{\phi}=\frac{\left(V_{N}-V_{\phi}\right)\left(V_{N}-V_{\phi}^{*}\right)\left(V_{N}+V_{\phi}\right)\left(V_{N}+V_{\phi}^{*}\right)}{\left(V-V_{\phi}\right)\left(V-V_{\phi}^{*}\right)\left(V+V_{\phi}\right)\left(V+V_{\phi}^{*}\right)} \\
\mathbf{W}_{\rho}=\frac{\left(W_{N}-W_{\rho}\right)\left(W_{N}-W_{\rho}^{*}\right)\left(W_{N}-1 / W_{\rho}\right)\left(W_{N}-1 / W_{\rho^{*}}\right)}{\left(W-W_{\rho}\right)\left(W-W_{\rho}^{*}\right)\left(W-1 / W_{\rho}\right)\left(W-1 / W_{\rho}^{*}\right)}
\end{gathered}
$$

Now, substituting instead of $P$ in (19) $\pi^{0}, \eta$ and $\eta^{\prime}$, we get three different unitary and analytic models for $F_{\gamma \pi^{0}}(t), F_{\gamma \eta}(t)$ and $F_{\gamma \eta^{\prime}}(t)$ respectively. They are applied to a description of existing data on $\pi^{0}, \eta$ and $\eta^{\prime}$ transition $\mathrm{FF}^{\prime}$ 's.

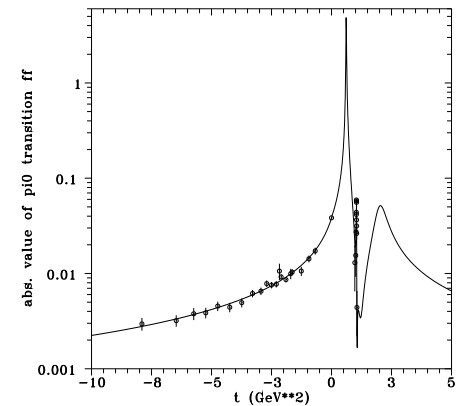

Figure 1: $\pi^{0}$ transition form factor.

$\pi^{0}$ : The best description (see Fig. 1 ) is achieved with $\chi^{2}=23.2$ i.e. $\chi^{2} / n d f=0.83$ and following values of parameters :

$$
\begin{aligned}
& t_{i n}^{s}=0.6081 G e V^{2} ; \quad t_{i n}^{v}=1.0464 G e V^{2} \\
& \left(f_{\omega \gamma \pi^{0}} / f_{\omega}\right)=0.1129 \pm 0.0007 \\
& \left(f_{\phi \gamma \pi^{0}} / f_{\phi}\right)=-0.0002 \pm 0.0001 \\
& \left(f_{\rho \gamma \pi^{0}} / f_{\rho}\right)=-0.0769 \pm 0.0007
\end{aligned}
$$

$\eta$ : The best description (see Fig. 2 ) is achieved with $\chi^{2}=47.5$ i.e. $\chi^{2} / n d f=1.01$ and following values of parameters : $t_{i n}^{s}=1.0323 \mathrm{GeV}^{2} ; \quad t_{i n}^{v}=1.5414 \mathrm{GeV}^{2}$ 


$$
\begin{aligned}
& \left(f_{\omega \gamma \eta} / f_{\omega}\right)=-.0524 \pm 0.0201 \\
& \left(f_{\phi \gamma \eta} / f_{\phi}\right)=-0.0013 \pm 0.0001 \\
& \left(f_{\rho \gamma \eta} / f_{\rho}\right)=0.0821 \pm 0.0202
\end{aligned}
$$

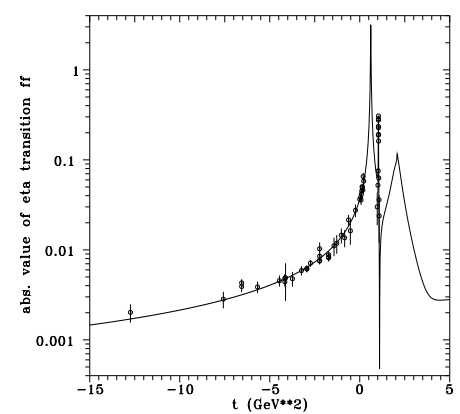

Figure 2: $\eta$ transition form factor.

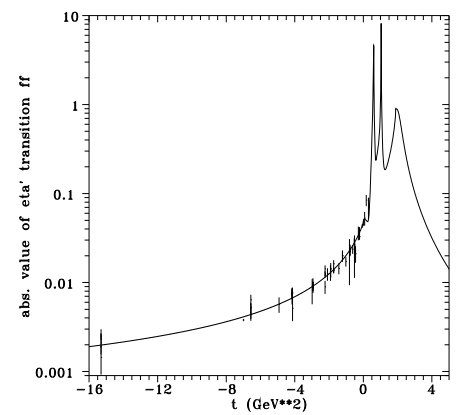

Figure 3: $\eta^{\prime}$ transition form factor.

$\eta^{\prime}$ : The best description (see Fig.3) is achieved with $\chi^{2}=67.4$ i.e. $\chi^{2} / n d f=1.25$ and following values of parameters :

$$
\begin{aligned}
& t_{i n}^{s}=1.0374 G e V^{2} ; \quad t_{i n}^{v}=1.5174 G e V^{2} \\
& \left(f_{\omega \gamma \eta^{\prime}} / f_{\omega}\right)=-.1623 \pm 0.0020 \\
& \left(f_{\phi \gamma \eta^{\prime}} / f_{\phi}\right)=0.1182 \pm 0.0052 \\
& \left(f_{\rho \gamma \eta^{\prime}} / f_{\rho}\right)=0.1253 \pm 0.0013
\end{aligned}
$$

\section{CONCLUSIONS}

We have briefly reviewed the present-day experimental and theoretical situation about the pseudoscalar meson transition FF's and came to the conclusion that there is no reliable prediction of them in the time-like region. Since there are besides quite good 
experimental information in the space-like region new Novosibirsk measurements of $e^{+} e^{-}$annihilation processes into $P \gamma$ at the $\phi$-meson peak, we have constructed the well founded unitary and analytic model of $F_{\gamma \pi^{0}}(t), F_{\gamma \eta}(t)$ and $F_{\gamma \eta^{\prime}}(t)$ with four vectormesons and predicted a reasonable behaviour of these transition FF's in the spacelike and time-like regions simultaneously. Results can be applied for a prediction of various cross-sections and decay rates in which pseudoscalar meson transition FF's appear, further, an evaluation of contributions of $e^{+} e^{-} \rightarrow \gamma P$ processes into the muon anomalous magnetic moment can be carried out and also the strange pseudoscalar meson transition FF's can be in principle predicted for the first time.

This work was in part supported by Slovak Grant Agency for Sciences, Grant 2/1111/22 (S.D. and R.P.) and Grant 1/7068/22 (A.Z.D.)

\section{References}

[1] S.J. Brodsky and G.P. Lepage, Phys. Rev. D 24 (1981) 1808.

[2] Ch. Berger et al, Phys. Lett. 142 B (1984) 125.

[3] H.-J. Behrend et al, Z. Phys. C49 (1991) 401.

[4] H. Aihara et al, Phys. Rev. Lett. 64 (1990) 172.

[5] J. Gronberg et al, Phys. Rev. D57 (1998) 33.

[6] M.N. Achasov et al, preprint Budker INP 99-39, Novosibirsk (1999).

[7] R.I. Djhelyadin et al, Phys. Lett. 94B (1980) 548.

[8] V.P. Druzhinin et al, Phys. Lett. 144B (1984) 136.

[9] S.I. Dolinsky et al, Z. Phys C42 (1989) 511.

[10] V.A. Viktorov et al, Sov. J. Nucl. Phys. 32 (1980) 520. 